\title{
IDENTIFICATION OF RISK FACTORS FOR DEATH FROM TETANUS IN PERNAMBUCO, BRAZIL: A CASE-CONTROL STUDY
}

\author{
Demócrito B. MIRANDA-FILHO, Ricardo A.A. XIMENES(1,2), Silvya N. BERNARDINO(1) \& Abelardo G. ESCARIÃO(1)
}

\begin{abstract}
SUMMARY
A case-control study was conducted to identify risk factors for death from tetanus in the State of Pernambuco, Brazil. Information was obtained from medical records of 152 cases and 152 controls, admitted to the tetanus unit in the State University Hospital, in Recife, from 1990 to 1995.

Variables were grouped in three different sets. Crude and adjusted odds ratios, p-values and 95\% confidence intervals were estimated. Variables selected in the multivariate analysis in each set were controlled for the effect of those selected in the others.

All factors related to the disease progression - incubation period, time elapsed between the occurrence of the first tetanus symptom and admission, and period of onset - showed a statistically significant association with death from tetanus. Similarly, signs and/or symptoms occurring on admission or in the following 24 hours (second set): reflex spasms, neck stiffness, respiratory signs/symptoms and respiratory failure requiring artificial ventilation (third set) were associated with death from tetanus even when adjusted for the effect of the others.
\end{abstract}

KEYWORDS: Tetanus; Death from tetanus; Risk factors; Disease progression; Case-control.

\section{INTRODUCTION}

It is estimated that there are approximately one million tetanus cases per year in the world, with a fatality rate varying from 20 to $50 \%{ }^{13}$, thus constituting an important public health problem, especially in many countries of Latin America, Africa, Asia and Oceania.

In Brazil the fatality rate has been around $30 \%$ for some years and in 1998 the accidental (non-umbilical) tetanus incidence coefficient was 4.65 per 1,000,000 inhabitants, with a total of 753 cases registered. In the state of Pernambuco the annual incidence rate in 1998 was 7.84 per $1,000,000^{10}$.

The handling of tetanus patients often raises doubts concerning prognosis. This has brought about several prognostic classifications derived from the identification of factors associated with death, with the aim of guiding the physician to anticipate complications through the adoption of more or less standardized measures for certain groups of patients. The information, which is generally used, concerns the rate at which the disease develops, together with clinical criteria. Although apparently well established, these criteria are far from being unanimously acclaimed by the various authors. Even when use is made of indicators that are widely known in medical literature, such as period of onset, incubation period, there is disagreement as to the cut-off points used in their categorisation ${ }^{1,3,6,16,17}$. Considering the reality of health care in developing countries, where the availability of beds in intensive care units is extremely limited or non-existent, the early identification of factors which have a key influence on the development of tetanus, especially regarding the risk of death, are of fundamental importance. In this paper we discuss those risk factors that may be considered as predictable factors of death from tetanus. We comment on those that may be identified in an early phase of the disease, i. e., on admission to hospital or within 24 hours of hospitalization, and on the complications and/or clinical occurrences displayed by tetanus patients which are associated with a higher mortality risk. One of the aspects in which this study differs from previous works is that the analysis of variables was carried out controlling for the effect of the others, either in the same group or in the other sets of variables, and the relative magnitude of the risk of death for individuals having various sets of values for the predictor variables was estimated. In this way, it is hoped to contribute with information which may facilitate or improve the planning of therapeutic procedures, especially for those patients at greatest risk and increase the efficiency of patient care through a greater co-ordination of resources, activities and efforts.

(1) Faculdade de Ciências Médicas, Universidade de Pernambuco-UPE, Recife, PE, Brasil.

(2) Departamento de Medicina Tropical da Universidade Federal de Pernambuco-UFPE, Recife, PE, Brasil.

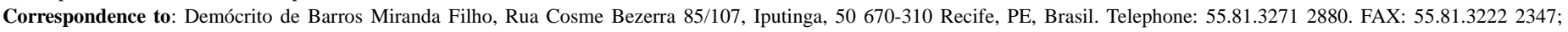
55.81.3221 2238 


\section{MATERIAL AND METHODS}

$\begin{array}{ll}\text { Glossary } & \\ \text { APN } & \text { apnea } \\ \text { ARF } & \text { acute renal failure } \\ \text { CARR } & \begin{array}{l}\text { cardiac arrhythmia } \\ \text { CI }\end{array} \\ \text { confidence limits } \\ \text { DISPH } & \text { Disphagia } \\ \text { HUOC } & \text { The Oswaldo Cruz University Hospital } \\ \text { IP } & \text { Incubation period } \\ \text { NCKS } & \text { Neck stiffness } \\ \text { OCC } & \text { Other cardiovascular complications } \\ \text { ONC } & \text { Other neurological complications } \\ \text { ORC } & \text { Other respiratory complications } \\ \text { PO } & \text { Period of onset } \\ \text { RFRAR } & \text { Respiratory failure requiring artificial respiration } \\ \text { RI } & \text { Respiratory infection } \\ \text { RSS } & \text { Respiratory signs/symptoms } \\ \text { SP24 } & \text { Spasms in the first } 24 \text { hours } \\ \text { TOAH } & \text { Time elapsed between the onset of symptoms and admission } \\ & \text { to hospital } \\ \text { UGB } & \text { Upper gastrointestinal bleeding }\end{array}$

Study Design: We used a case-control methodology. All the patients admitted to the Infectious and Parasitic Diseases Service of the HUOC (The Oswaldo Cruz University Hospital) clinically diagnosed as having tetanus and that subsequently died as a result of the disease or associated causes were considered as cases, whilst those with the same diagnosis but that were subsequently discharged were considered as controls. A study of medical records of tetanus patients admitted to the HUOC between 1990 and 1995 was carried out. We selected all cases, i. e., 152, that met the criteria described and 152 controls out of 333 tetanus patients who survived. To avoid selection bias, especially among those used as controls, the non-cases were divided into six strata, corresponding to the year of hospital admission, and a simple random sample taken from each of these strata, keeping the same proportional distribution as the cases. It should be stressed that the HUOC is the only hospital which is reference for tetanus treatment in the State of Pernambuco. Twelve cases diagnosed as having tetanus on admission but that subsequently developed little or uncharacteristically, or that died soon after admission (in such a way as to cast doubt on the final diagnosis) or those that left hospital for any reason other than medical discharge were excluded from the study. Some precautions were taken to minimize the possibility of information bias. Since the information utilized had been previously documented in the form of medical records, the risk of bias due to memory, a particular problem in case-control studies, was eliminated. Classification errors may occur in relation to the effect or exposure. Death being the effect in question in this work, the possibility of classification error regarding effect is practically zero. Although the possibility of error concerning exposure cannot be ruled out, the group (case or control) to which each patient belonged was unknown both on admission and during evolution. It is therefore likely that their distribution occurred randomly (or non-differentially), which would imply that the estimate of the association measure (odds ratio) would tend to that of the null hypothesis. An information bias could also have occurred on collecting data from the records, if the researchers had differentially assessed and registered information concerning each group, resulting in a differential classification error. In order to avoid such problems, information was collected by using a standard form with objective yes/ no and coded response questions. In spite of the fact that there was a standard treatment that should be applied to all patients there may have been individual differences that may have affected the outcome. However differences in treatment were not dependent on the category of exposure, so we would not expect that treatment would confound these associations, for a variable to confound a relationship must be associated with both the exposure and the outcome. The same reasoning may be applied to underlying diseases. It would be expected that they could affect the outcome but not that they would vary according to the category of the other factors (exposures).

Sample: To calculate the sample size we used the following parameters: $\alpha=5 \%, \beta=20 \%$ (power $=80 \%$ ); to estimate the proportion of exposure among controls (and cases) we used, as reference, the values obtained by ARMITAGE \& CLIFFORD" 1 on two variables: "spasms on admission" and "time elapsed between onset of symptoms and admission to hospital".

The sample size estimated was 190 patients, 95 in each group. To be able to adjust for confounding effects, we worked with a total of 304 patients in the ratio of one case to one control.

Data Analysis: For the purpose of analysis, the variables were grouped in three sets, each containing sex, age and the dependent variable, in addition to the identification and specific variables of the set. In the initial phase of analysis, the association of each variable with death from tetanus was verified. The magnitude of this association was expressed through the odds ratio. The statistical significance of the results obtained was tested using a $95 \%$ confidence interval and the qui square test with Yates correction where appropriate, a value of $\mathrm{p}<0.05$ being considered significant.

From the identification of the variables whose association with death from tetanus was statistically significant in the univariate analysis, a multivariate analysis was carried out using multiple logistic regression. The model was initially saturated with the inclusion of all the variables in each set. The removal of each variable from the proposed model was tested (maximum likelihood test) and expressed as a value of "p" (method "bstep(lr)" - SPSS PC). In the analyses with two or more independent variables, those variables with more than three categories were regrouped to give results with greater statistical stability. Finally, the variables which were retained in the final model in each set were adjusted for the effect of the others.

For the calculation of sample size, univariate analysis and data preparation for multivariate analysis we used the Epi-Info (version 5) program and for the logistic regression, the SPSS-PC.

\section{RESULTS}

General Characteristics of the Sample: Of the 304 patients studied, 232 were male, accounting for slightly more than $75 \%$ of the sample, a ratio of more than $3: 1$ in relation to females.

Biological Variables (age and sex) and Death from Tetanus: Taking the 12-20 age group as reference, an association between age group and tetanus death was verified, the difference being statistically 


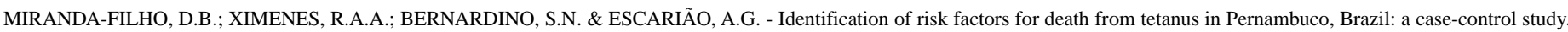
Rev. Inst. Med. trop. S. Paulo, 42(6):333-339, 2000.

significant between the reference level and the two others. Concerning sex, the risk of death was almost twice as great for female patients, this difference being statistically significant (Table 1).

When age and sex variables were adjusted for each other by means of logistic regression, the association remained statistically significant only with age. As it was a potential confounding factor, this variable was included in the other analysis of non-biological variables.

The First Set of Variables - Related to Tetanus Progression: All variables analyzed in this group: the time elapsed between the onset of symptoms and admission to hospital (TOAH), incubation period (IP) and period of onset (PO) showed a statistically significant association with tetanus death in the univariate analysis (data not shown). Therefore they were introduced in a multivariate model and all variables were kept in the final model (Table 2). Patients who were admitted to the hospital within the first 36 hours after the onset of symptoms, as well as those with either a shorter incubation period or a shorter period of onset, had a worse prognosis, the magnitude of the odds ratios varying with the category considered.

The Second Set of Variables - Signs and Symptoms on Admission or in the First 24 Hours: In this group, each variable has only two categories ("yes" or "no"), according to the presence or absence of certain signs/symptoms in the first $24 \mathrm{~h}$ of hospitalization. The absence of signs/ symptoms considered was taken as the reference level. In the univariate analysis there was a statistically significant association between disphagia (DISPH), spasms in the first 24h (SP24), neck stiffness (NCKS), respiratory signs/symptoms (RSS) (at least one of the following: tachypnea, dyspnea, apnea attacks, rhonchi or crepitations detected by stethoscope, cyanosis, cough, bronchial spasms, pleuritic pain) and death from tetanus. However, statistically significant association was not observed concerning spasms on admission, paraspinal muscular stiffness (thoracic and lumbar), abdominal stiffness, fever and infection at point of entry in patients and the risk of death from tetanus (data not shown).

For multivariate analysis of the second set of variables, those that had showed a statistically significant association with tetanus death were considered: DISPH, SP24, NCKS and RSS. All remained in the final model. The introduction of the variable age into the model leads to the removal of the variable DISPH from the final model (maximum likelihood ratio statistic $=0.17$, d.f. $=1$ and p>0.05), whilst keeping the others (Table 3).

The Third Set of Variables - Complications and Death from Tetanus: In this block, like in the one above described, all the variables have only two categories (yes and no). From the univariate analysis (data not shown) there was a statistically significant association between death from tetanus and respiratory infection (RI), respiratory failure requiring artificial respiration (RFRAR), apnea (APN), and "other respiratory complications" - ORC (i.e., the presence of at least one of the following: bronchospasm, hydrothorax, post tracheotomy bleeding, prolonged hypoxia, pulmonary embolization, tracheoesofagic fistula, empiema). A statistically significant association was also found between death from tetanus and cardiac arrhythmia (CARR) and "other cardiovascular complications" - OCC (one or more of the manifestations listed: cardiorespiratory arrest, shock, congestive heart failure, hypertensive crises, phlebitis, acute pulmonary edema, acute myocardial infarction). Similarly, an increased risk of death was observed for those patients
Table 1

Crude odds ratio for the association of death from tetanus with age and sex

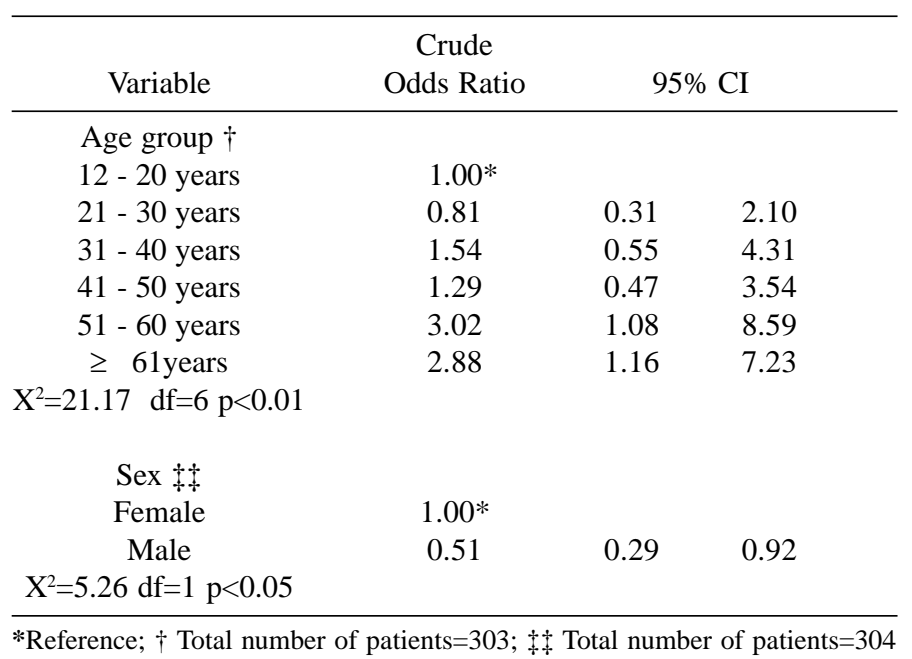

Table 2

Adjusted odds ratios, confidence intervals and "p" values for the association of death from tetanus with time elapsed between the onset of symptoms and admission to hospital (TOAH), incubation period (IP), period of onset (PO) and age group

\begin{tabular}{ccccc}
\hline Variable & Odds Ratio & \multicolumn{2}{c}{$95 \%$ CI } & $\mathrm{p}$ value \\
\hline age group & & & & \\
12 - 30 years & $1.00^{*}$ & & & \\
31 - 50 years & 2.44 & 1.06 & 5.61 & $\mathrm{p}<0.05$ \\
$\geq 51$ years & 5.05 & 2.23 & 11.42 & $\mathrm{p}<0.001$ \\
TOAH & & & & \\
$0-36 \mathrm{~h}$ & $1.00^{*}$ & & & \\
$37-72 \mathrm{~h}$ & 0.48 & 0.20 & 1.14 & $\mathrm{NS}$ \\
$\geq 73 \mathrm{~h}$ & 0.21 & 0.08 & 0.56 & $\mathrm{p}<0.01$ \\
IP & & & & \\
$1-$ 6 days & $1.00^{*}$ & & & \\
7 - 10 days & 0.92 & 0.41 & 2.04 & $\mathrm{NS}$ \\
$\geq 11$ days & 0.32 & 0.14 & 0.75 & $\mathrm{p}<0.01$ \\
PO & & & & \\
0 - $36 \mathrm{~h}$ & $1.00^{*}$ & & & \\
$37-48 \mathrm{~h}$ & 0.52 & 0.20 & 1.36 & $\mathrm{NS}$ \\
$49-72 \mathrm{~h}$ & 0.16 & 0.03 & 0.84 & $\mathrm{p}<0.001$ \\
$\geq 73 \mathrm{~h}$ & 0.41 & 0.15 & 1.17 & $\mathrm{NS}$ \\
\hline
\end{tabular}

* Reference; Total number of patients $=216$

with upper gastrointestinal bleeding (UGB) and acute renal failure (ARF). The odds ratio for those with "other neurological complications" (ONC) was not significantly different from that of the baseline level; by ONC we mean the presence of at least one of the following: coma, diplopia, facial paresthesia, stroke, vertebral-basilar dysfunction (data not shown).

The effect of each variable selected in the univariate analysis was then controlled for the others belonging to the same system and for age. 


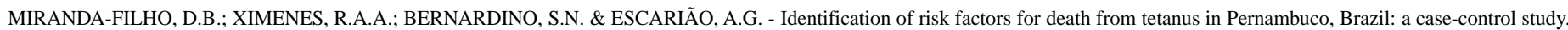
Rev. Inst. Med. trop. S. Paulo, 42(6):333-339, 2000.

Table 3

Adjusted odds ratios, confidence intervals and "p" values for the association of death from tetanus with disphagia (DISPH), spasms in the first 24h (SP24), neck stiffness (NCKS), respiratory signs/symptoms (RSS) and age group

\begin{tabular}{lcccc}
\hline Variables & Odds Ratio & \multicolumn{2}{c}{$95 \%$ CI } & $\mathrm{p}$ value \\
\hline Age group & & & & \\
12 - 30 years & $1.00^{*}$ & & & \\
31 - 50 years & 1.95 & 1.02 & 3.71 & $\mathrm{p}<0.05$ \\
$\geq$ 51 years & 3.51 & 1.89 & 6.51 & $\mathrm{p}<0.001$ \\
SP24 & & & & \\
No & $1.00^{*}$ & & & \\
Yes & 4.54 & 2.25 & 9.16 & $\mathrm{p}<0.001$ \\
NCKS & & & & \\
No & $1.00^{*}$ & & & \\
Yes & 2.51 & 1.27 & 4.98 & $\mathrm{p}<0.01$ \\
RSS & & & & \\
No & $1.00^{*}$ & & & $\mathrm{p}<0.01$ \\
Yes & 2.72 & 1.45 & 5.11 & \\
\hline
\end{tabular}

* Reference; Total number of patients $=301$

Table 5

Adjusted odds ratios, confidence intervals and "p" values for the association of death from tetanus with time elapsed between the onset of symptoms and admission to hospital (TOAH), incubation period (IP), period of onset (PO), neck stiffness (NCKS), spasms in the first 24h (SP24) and respiratory signs/ symptoms (RSS) - set 1 and 2 variables

\begin{tabular}{ccccc}
\hline Variables & Odds Ratio & \multicolumn{2}{c}{$95 \%$ CI } & $\mathrm{p}$ value \\
\hline TOAH & & & & \\
$0-36 \mathrm{~h}$ & $1.00^{*}$ & & & \\
$37-72 \mathrm{~h}$ & 0.39 & 0.36 & 0.92 & $\mathrm{p}<0.05$ \\
$\geq 73 \mathrm{~h}$ & 0.12 & 0.04 & 0.36 & $\mathrm{p}<0.001$ \\
IP & & & & \\
$1-$ days & $1.00^{*}$ & & & \\
$7-10$ days & 0.99 & 0.45 & 2.16 & $\mathrm{NS}$ \\
$\geq 11$ days & 0.30 & 0.13 & 0.68 & $\mathrm{p}<0.01$ \\
PO & & & & \\
$0-36 \mathrm{~h}$ & $1.00^{*}$ & & & \\
$37-48 \mathrm{~h}$ & 0.63 & 0.23 & 1.69 & $\mathrm{NS}$ \\
$49-72 \mathrm{~h}$ & 0.31 & 0.11 & 0.85 & $\mathrm{p}<0.05$ \\
$\geq 73 \mathrm{~h}$ & 1.18 & 0.39 & 3.58 & $\mathrm{NS}$ \\
NCKS & & & & \\
No & $1.00^{*}$ & & & \\
Yes & 2.28 & 0.94 & 5.54 & $\mathrm{NS} \quad(\mathrm{p}=0.069)$ \\
SP24 & & & & \\
No & $1.00^{*}$ & & & \\
Yes & 2.69 & 1.00 & 7.21 & $\mathrm{p}<0.05$ \\
RSS & & & & \\
No & $1.00^{*}$ & & & \\
Yes & 2.37 & 1.22 & 5.22 & $\mathrm{p}<0.05$ \\
\hline
\end{tabular}

* Reference; Total number of patients $=216$
Table 4

Adjusted odds ratios, confidence intervals and "p" values for the association of death from tetanus with respiratory failure requiring artificial respiration (RFRAR), apnea (APN), cardiac arrhythmia (CARR), "other cardiovascular complications" (OCC), acute renal failure (ARF) and upper gastrointestinal bleeding (UGB)

\begin{tabular}{ccccc}
\hline Variables & Odds Ratio & \multicolumn{2}{c}{$95 \% \mathrm{CI}$} & $\mathrm{p}$ value \\
\hline RFRAR & & & & \\
No & $1.00 *$ & & & \\
Yes & 16.22 & 8.33 & 31.58 & $\mathrm{p}<0.001$ \\
APN & & & & \\
No & $1.00 *$ & & & \\
Yes & 3.75 & 1.75 & 8.05 & $\mathrm{p}<0.01$ \\
ARF & & & & \\
No & $1.00 *$ & & & \\
Yes & 2.55 & 1.12 & 5.80 & $\mathrm{p}<0.05$ \\
\hline
\end{tabular}

*Reference; Total number of patients $=300$

Table 6

Adjusted odds ratios, confidence intervals and "p" values for the association of death from tetanus with time elapsed between the onset of symptoms and admission to hospital (TOAH), incubation period (IP) and period of onset (PO), neck stiffness (NCKS), spasms in the first 24h (SP24), respiratory signs/ symptoms (RSS), respiratory failure requiring artificial respiration (RFRAR), apnea (APN), acute renal failure (ARF) - set 1,2 and 3 variables

\begin{tabular}{ccccc}
\hline Variables & Odds Ratio & \multicolumn{2}{c}{$95 \%$ CI } & $\mathrm{p}$ value \\
\hline TOAH & & & & \\
$0-36 \mathrm{~h}$ & $1.00^{*}$ & & & \\
$37-72 \mathrm{~h}$ & 1.27 & 0.72 & 2.23 & $\mathrm{NS}$ \\
$\geq 73 \mathrm{~h}$ & 0.34 & 0.17 & 0.67 & $\mathrm{p}<0.01$ \\
IP & & & & \\
$1-$ 6 days & $1.00^{*}$ & & & \\
$7-10$ days & 1.31 & 0.76 & 2.26 & $\mathrm{NS}$ \\
$\geq 11$ days & 0.47 & 0.27 & 0.83 & $\mathrm{p}<0.01$ \\
PO & & & & \\
$0-36 \mathrm{~h}$ & $1.00^{*}$ & & & \\
$37-48 \mathrm{~h}$ & 0.88 & 0.42 & 1.87 & $\mathrm{NS}$ \\
$49-72 \mathrm{~h}$ & 0.41 & 0.19 & 0.89 & $\mathrm{p}<0.05$ \\
$\geq 73 \mathrm{~h}$ & 2.25 & 1.02 & 4.92 & $\mathrm{p}<0.05$ \\
NCKS & & & & \\
No & $1.00^{*}$ & & & $\mathrm{p}<0.05$ \\
Yes & 1.72 & 1.03 & 2.87 & \\
RFRAR & & & & $\mathrm{p}<0.001$ \\
No & $1.00^{*}$ & & & \\
Yes & 4.43 & 2.95 & 6.66 & \\
\hline
\end{tabular}

*Reference; Total number of patients $=215$ 
A multivariate analysis was conducted using the respiratory complications (RI, RFRAR, APN, and ORC) that showed a statistically significant association with death from tetanus in the univariate analysis. Using a step-down procedure, the variables RFRAR and APN were retained in the final model. After adjusting RFRAR and apnea for age, the final model maintains the same variables, suggesting that the association of these variables with death is independent of age group (data not shown).

The adjustment of the complications related to the cardio-vascular system (CARR and OCC) for each other and for age suggests that the association of each one of the mentioned variables with tetanus death is independent of the others (data not shown).

Urinary (acute renal failure - ARF) and digestive (upper gastrointestinal bleeding - UGB) tract complications were adjusted separately for age. In both cases the analysis showed statistically significant association with tetanus death independent of the age of the patient (data not shown).

From a model saturated with all the variables of the block selected in the previous phases, multivariate analysis was carried out. The removal of the variables CARR, OCC and UGB from the model was not statistically significant, such that only the variables RFRAR, apnea and ARF remained in the model (Table 4).

Multiple Logistic Regression with Selected Set 1 and Set 2 Variables: In order to verify which variables could best be considered for the early identification of patients with a greater risk of tetanus death, those which can be obtained in the first 24 hours of hospitalization (TOAH, IP, PO, NCKS, SP24 and RSS) were introduced into a multivariate model (Table 5).

The results show that the magnitude of the estimated odds ratios remained practically unchanged, suggesting a minimum confounding effect, if any, among the variables.

Multiple Logistic Regression of "Progression Variables" (Set 1) and with Signs and Symptoms on Admission or During the First 24 Hours (Set 2) and Complications (Set 3): We introduced into a multivariate model the set of variables related to the speed of tetanus progress on admission or during the first 24 hours and selected in the multivariate analysis and the complications, that is: TOAH, IP, PO, NCKS, SP24, RSS, RFRAR, APN and ARF. No statistically significant difference was found with the removal of four (SP24, RSS, APN and ARF) of the nine variables under analysis. The other variables remained in the final model suggesting a closer association with tetanus death (Table 6).

\section{DISCUSSION}

In the analysis of biological factors, it was found that patients over 51 years of age show a risk of tetanus death approximately three times greater when compared with those between 12 and 20.

The association between sex and tetanus death became non significant when controlled for age. Death from tetanus is proportionally greater in the elderly, irrespective of sex. Our sample contains a higher proportion of elderly women in relation to younger ones, and consequently a greater proportion of tetanus death, suggesting a higher mortality risk for females.
It is likely that younger women, owing to their superior coverage in terms of vaccination because of the prioritization of pre-natal vaccination as a neonatal tetanus eradication policy, are less prone to the disease; for this reason the incidence of tetanus has been much greater in women above reproductive age.

Concerning the variables which map the progression of tetanus (progression variables), our results show that all may be considered as predicting a higher risk of tetanus death. When shorter, the time elapsed between the onset of symptoms and admission to hospital (TOAH $\leq 72 \mathrm{~h}$ ), the incubation period (IP $\leq$ 10days) and period of onset $(\mathbf{P O} \leq \mathbf{4 8 h})$ may suggest faster and more aggressive evolution of the disease, implying a worse prognosis. Regarding the period of onset there was no statistically significant difference between the odds ratio estimated for the category $\mathbf{P O} \geq \mathbf{7 3 h}$ and reference level. This finding was not observed in the univariate analysis $(\mathbf{P O} \geq \mathbf{7 3 h}$ : odds ratio $0.16,95 \% \mathrm{Cl}$ : $0.08-0.33 \mathrm{p}<0.001$ ) and may be due to a smaller sample size in the multivariate analysis and to the correlation between variables. The majority of authors consulted consider the use of incubation and onset periods as suggestive prognostic criteria, shorter periods being indicative of poor prognosis $3,4,6,8,9,15,17$.

Our results support the findings of ARMITAGE \& CLIFFORD ${ }^{1}$, on showing the association between short IP and TOAH and death from tetanus. It is evident that the observation of TOAH as an early prognostic indicator could be of great relevance in the therapeutic orientation of tetanus patients, especially because it is easy to obtain in early phases of the disease. In our study it has shown itself to be a variable with important mortality-predicting power both in univariate analysis and in all phases of the multivariate analysis.

Signs and symptoms have generally been used in clinical classifications, being less frequently associated with prognosis. Concerning the presence of spasms on admission, in contrast to ARMITAGE \& CLIFFORD $^{1}$, we have not found significant association with mortality, although the association became significant when considering the presence of spasms in the first $\mathbf{2 4}$ hours after admission (SP24). It is possible that this is due to faults in the description of the physical examination on admission, when the registration of spasms could have been omitted.

With the mutual adjustment of the variables selected in set 1 and 2, six - TOAH, IP, PO, SP24, NCKS and RSS - remained in the final model, showing that the association of each one with mortality is independent of the others. These six variables therefore form a set of information which, when obtained either on admission to hospital or in the subsequent 24 hours, can be used to identify subgroups with a greater risk of death from tetanus. In our literature review, however, the association between neck stiffness and death from tetanus was not reported. Several clinical classifications of the severity of tetanus use the presence and intensity of disphagia ${ }^{2,9,14,17}$. In our study this association was not confirmed after adjustment for age, although in this evaluation we have not graduated the extent to which swallowing had been implicated. It is necessary to take into account, however, that the description of neck stiffness generally refers to the contraction of the muscles behind the neck with the limitation or restriction of head movement. In the case of tetanus this postural stiffness can be accompanied by the contraction of other groups of neck muscles. This 
restriction may interfere in swallowing movements and/or in the "protection" of the air passages against the aspiration of secretions. In this particular case the hyperextension of the neck may contribute to bronchial problems and increase the risk of death. From this point of view, disphagia may be part of a group of factors which make up the neck stiffness variable and which may, in a sense, be associated with mortality by allowing the appearance of a greater number of complications.

The RSS variable corresponds to a set of respiratory signs and symptoms cited in the medical registers of several patients, generally during their examination on admission, which could be considered as variables in isolation. On analyzing the data, we do not give special weight to any type of sign or symptom, but we should consider the possibility of there being differences between each component of the set insofar as their potential association with tetanus mortality. We therefore consider the respiratory findings together in predicting death from tetanus.

Identifying which complications usually precede the death of tetanus patients may provide useful information, both in the guidance of future therapy programmes and in the re-evaluation of the structure of treatment for tetanus patients.

It can be concluded that respiratory failure requiring artificial respiration (RFRAR) is still the complication most strongly associated with the risk of death among our patients. The frequent occurrence of respiratory infections in these individuals is a contributory factor in the prognosis, since the majority of cases are caused by hospital germs ${ }^{6}$. To this may be added the fact that these patients are treated in intermediate care units, when they should be in intensive care. In recent publications on the treatment of tetanus in developed countries, respiratory complications are still cited as the most frequent, although they describe a trend towards a reduction in their contribution as the main cause of death following advances in the care of patients undergoing prolonged artificial respiration ${ }^{4,7}$.

Apnea attacks can be triggered by several mechanisms which may act in conjunction or isolatedly. The occurrence of subintrant spasms and excessive contraction of the thorax, hyperactivity or malfunction of the sympathetic nervous system, hypoxia, localized glottal spasms or even the excess of sedation used to control spasms $s^{4,6,12}$. It can easily be seen that this type of complication occurs in the most serious forms of the disease, a fact that would already explain the greater frequency of deaths in these individuals, contributing to or even being responsible for the fatal outcome.

Acute renal failure may be caused by more than one mechanism like rhabdomyolysis ${ }^{4,6}$, the use of nephrotoxic drugs, dehydration and neurogenic bladder ${ }^{8}$. The identification of IRA as a factor associated with death of tetanus patients is important since it allows prophylactic action. The careful replacement of liquids and electrolytes, the appropriate choice of drugs in the handling of high-risk patients and appropriate control of spasms can all contribute to the reduction of this complication.

In the multiple logistic regression analysis with the variables of the three blocks cited all the "progression variables" (TOAH, IP and OP), one variable from the second block (cervical stiffness) and one of the complications (RFRAR) remained in the final model. This indicates a strong association of the first ones with tetanus death, suggesting their predictive potential concerning the prognosis of the disease. The exclusion of the other variables from this final model, however, does not mean that their association with tetanus death is non-existent or unimportant. It is worth pointing out that there is a correlation among these variables and that those selected in the final model are the ones that have the closest association with tetanus death and therefore have an important predictive potential, independently of the others.

From our findings we would like to emphasize the following points: First of all, the concern for early identification of risk factors for tetanus death in this type of patient may be of great value in guiding the adoption of early intervention measures and intensive care for high-risk subgroups. This data, besides being very helpful in the management of patients, may increase the service efficiency, contributing to provide optimal service within limited resources. Secondly, the methodology utilized enabled the risk of tetanus death to be quantified, i.e., the magnitude of the association was estimated for each factor studied. The case-control study also allowed each risk factor to be adjusted for the effect of the others either in the same group or in the other sets of variables, achieving a better understanding of the interrelationships among each variable, the outcome and additional factors, minimizing the problem of confounding factors among the variables studied. Third, finally, because this study has not only ratified the importance of certain factors already recognized in the literature as being important in determining the tetanus patient's prognosis $\left(\mathrm{age}^{11}\right.$, short incubation and progression periods of onset $^{1,3,4,5,6,16,17}$, presence of spasms in the first 24 hours $^{1}$ ), but also pointed out other prognostic indicators as yet little used (time elapsed between onset of symptoms and admission to hospital ${ }^{1}$ ) or even unmentioned in the literature consulted (occurrence of neck stiffness in the first 24 hours and the occurrence of respiratory signs and symptoms in the first 24 hours of hospitalization, such as the presence of at least one of the following: tachypnea, dyspnea, attacks of apnea, rhonchi or respiratory crepitations, cyanosis, cough, bronchial spasms, pleuritic pain).

Finally, it is important to point out that, even after adjusting for complications, the progression variables remain their ability to predict death risk. This reinforces the importance of these variables for the early identification of higher risk subgroups. In addition, it suggests that there may be other mechanisms related to determination of death in tetanus patients, that is, perhaps the poisoning itself and its immediate effects may be directly responsible for some of the deaths among the patients studied. It is therefore necessary that new studies are carried out with a view to the treatment of poisoning in tetanus. A re-evaluation of clinical and/or prognosis classification criteria from tetanus patients, useful both in planning therapy and in the evaluation of results, will be able to make use of some of the information discussed here.

\section{RESUMO}

\section{Identificação de fatores de risco para morte por tétano em Pernambuco, Brasil: um estudo de caso-controle}

Um estudo de caso-controle foi conduzido no Estado de Pernambuco, Brasil, para identificar fatores de risco para morte por tétano. As informações foram obtidas dos prontuários médicos de 152 casos e 152 controles, admitidos na Unidade de Tétano do Hospital Universitário Oswaldo Cruz, na cidade do Recife, de 1990 a 1995. 
As variáveis foram agrupadas em três diferentes conjuntos. Foram estimados os "odds ratios" brutos e ajustados, o intervalo de confiança de $95 \%$ e o valor de " $p$ ". As variáveis selecionadas na análise multivariada em cada conjunto, foram controladas para os efeitos daquelas selecionadas nos demais.

Todos os fatores relacionados à progressão da doença - período de incubação, tempo decorrido entre o primeiro sintoma de tétano e a admissão no hospital, e período de progressão - mostraram uma associação estatisticamente significante com morte por tétano. De modo semelhante, o segundo conjunto de variáveis - sinais e/ou sintomas que ocorreram à admissão ou nas primeiras 24 horas: espasmos, rigidez de nuca, sinais e sintomas respiratórios - bem como as variáveis do terceiro conjunto - insuficiência respiratória que necessitou de respiração artificial foram associadas com morte por tétano, mesmo quando ajustadas com os efeitos das demais variáveis.

\section{REFERENCES}

1. ARMITAGE, P. \& CLIFFORD, R. - Prognosis in Tetanus. Use of data from therapeutic trials. J. infect. Dis., 138: 1-8, 1978.

2. BETANCUR, J.; GOMEZ, L. \& CASTELlANOS, R. - Tetanos. Acta méd. colomb., 12: 289-293, 1987.

3. BLECK, T.P. - Clostridium tetani. In: MANDELL, G.L.; BENNETT, J.E. \& DOLIN, R. - Mandell, Douglas and Bennett's principles and practice of Infectious Diseases. 4. ed. New York, Churchill Livingstone, 1995. p. 2173-2178.

4. BLECK, T.P. - Tetanus: pathophysiology, management, and prophylaxis. Dis. Month, 37: 545-603, 1991.

5. COLE, L. \& YOUNGMAN, H. - Treatment of tetanus. Lancet, 1: 1017-1019, 1969.

6. DEBORD, T. \& LAPEYRE, E. - Tétanos. In: Encyclopédie Médico-Chirurgicale Maladies infectieuses. Paris, S.G.I.M.; Brugières, 1995. p.1-6, 8-038-G-10.
7. EDMONDSON, R.S. \& FLOWERS, M.W. - Intensive care in tetanus: management, complications and mortality in 100 cases. Brit. med. J., 1: 1401-1404, 1979.

8. HINRICHSEN, S.L. - Tétano. Rev. bras. Clín. Terap., 18: 449-463, 1989.

9. KOBEL, T. \& MARTI, M.C. - 100 ans aprés - Découverte du bacille du tétanus (1984). Rev. med. Suisse rom., 105: 547-556, 1985.

10. MINISTÉRIO DA SAÚDE - Dados referentes à situação epidemiológica do tétano acidental no Brasil. Brasilia, Ministério da Saúde, 1995. p. 1-10.

11. NOLLA-SALAS, M. \& GARCÉS-BRUSÉS, J. - Severity of tetanus in patients older than 80 years: comparative study with younger patients. Clin. infect. Dis., 16: 591$592,1993$.

12. PASTERNAK, J. - Tétano e outras doenças causadas por clostrídios. In: AMATO NETO V. \& BALDY, J.L.S. - Doenças transmissíveis. 3. ed. São Paulo, Sarvier, 1989. p. $811-825$

13. SANFORD, J.P. - Tetanus. Forgotten but not gone. New Engl. J. Med., 332: 812-813, 1995

14. SHARMA, N.; TRUBUHOVICH, R. \& THOMAS, M.G. - Tetanus in Auckland: a preventable disease. N. Z. med. J., 107: 82-84, 1994

15. TAVARES, W. - Infecções e trauma. I. Tétano. In: SCHECHTER, M. \& MARANGONI, D.V., ed. Doenças infecciosas: condutas diagnóstica e terapêutica. Rio de Janeiro, Guanabara Koogan, 1994. p. 387-392.

16. UDWADIA, F.E.; LALL, A.; UDWADIA, Z.F.; SEKHAR, M. \& VORA, A. - Tetanus and its complications: intensive care and management experience in 150 Indian patients. Epidem. Infect., 99: 675-684, 1987.

17. VERONESI, R.; FOCACCIA, R.; TAVARES, W. \& MAZZA, C.C. - Tétano. In: VERONESI, R. \& FOCACCIA, R., ed. Veronesi Tratado de Infectologia. São Paulo, Atheneu, 1997. p. 887-913.

Received: 16 May 2000

Accepted: 19 July 2000 